\title{
An evaluation of outcomes in patients with traumatic brain injury at a referral hospital in Tanzania: evidence from a survival analysis
}

\author{
Cyrus Elahi, MScGH, ${ }^{1,2}$ Thiago Augusto Hernandes Rocha, PhD, ${ }^{1,3}$ Núbia Cristina da Silva, PhD, ${ }^{1,4}$ \\ Francis M. Sakita, MD, ${ }^{5}$ Ansbert Sweetbert Ndebea, MD, ${ }^{5}$ Anthony Fuller, MD, MScGH, ${ }^{1,2}$ \\ Michael M. Haglund, MD, PhD, MACM, ${ }^{1,2}$ Blandina T. Mmbaga, MD, ${ }^{5}$ \\ João Ricardo Nickenig Vissoci, PhD, ${ }^{1,2,6}$ and Catherine A. Staton, MD, MScGH ${ }^{1,2,6}$
}

\begin{abstract}
${ }^{1}$ Division of Neurosurgery and Neurology, Department of Neurosurgery, Duke University Medical Center; ${ }^{2}$ Duke Global Health Institute, Duke University, Durham, North Carolina; ${ }^{3}$ Pan American Health Organization, World Health Organization, Brasilia; ${ }^{4}$ Methods Analytics and Technology for Health (MATH) Consortium, Belo Horizonte, Brazil; ${ }^{5 K i l i m a n j a r o ~ C h r i s t i a n ~ M e d i c a l ~ C e n t e r, ~}$ Moshi, Tanzania; and ${ }^{6}$ Division of Emergency Medicine, Department of Surgery, Duke University Medical Center, Durham, North Carolina
\end{abstract}

\begin{abstract}
OBJECTIVE The purpose of this study was to determine if patients with traumatic brain injury (TBI) in low- and middleincome countries who receive surgery have better outcomes than patients with TBI who do not receive surgery, and whether this differs with severity of injury.

METHODS The authors generated a series of Kaplan-Meier plots and performed multiple Cox proportional hazard models to assess the relationship between TBI surgery and TBI severity. The TBI severity was categorized using admission Glasgow Coma Scale scores: mild $(14,15)$, moderate $(9-13)$, or severe (3-8). The authors investigated outcomes from admission to hospital day 14. The outcome considered was the Glasgow Outcome Scale-Extended, categorized as poor outcome (1-4) and good outcome (5-8). The authors used TBI registry data collected from 2013 to 2017 at a regional referral hospital in Tanzania.
\end{abstract}

RESULTS Of the final 2502 patients, $609(24 \%)$ received surgery and $1893(76 \%)$ did not receive surgery. There were significantly fewer road traffic injuries and more violent causes of injury in those receiving surgery. Those receiving surgery were also more likely to receive care in the ICU, to have a poor outcome, to have a moderate or severe TBI, and to stay in the hospital longer. The hazard ratio for patients with TBI who underwent operation versus those who did not was $0.17(95 \% \mathrm{Cl} 0.06-0.49 ; p<0.001)$ in patients with moderate TBI; $0.2(95 \% \mathrm{Cl} 0.06-0.64 ; p=0.01)$ for those with mild $\mathrm{TBI}$, and 0.47 (95\% $\mathrm{Cl} 0.24-0.89 ; p=0.02)$ for those with severe TBI.

CONCLUSIONS Those who received surgery for their TBI had a lower hazard for poor outcome than those who did not. Surgical intervention was associated with the greatest improvement in outcomes for moderate head injuries, followed by mild and severe injuries. The findings suggest a reprioritization of patients with moderate TBI-a drastic change to the traditional practice within low- and middle-income countries in which the most severely injured patients are prioritized for care. https://thejns.org/doi/abs/10.3171/2019.7.FOCUS19316

KEYWORDS brain injuries; survival analysis; developing countries; critical care outcomes

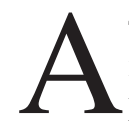
т an estimated 69 million cases annually, traumatic brain injury (TBI) is a devastating global health pathology with a growing burden of disease. ${ }^{7}$ This burden disproportionately occurs in low- and middleincome countries (LMICs), where the incidence of TBI is nearly 3 times the incidence in high-income countries (HICs). ${ }^{7}$ In sub-Saharan Africa (SSA), an estimated 801 people per 100,000 suffer TBI, with in-hospital mortality rates as high as $47 \%$ for severe TBI. ${ }^{22}$ In HICs, neurosurgical interventions have been shown to prevent morbidity

ABBREVIATIONS CoxPH = Cox proportional hazard model; GCS = Glasgow Coma Scale; GOS = Glasgow Outcome Scale; GOSE = Glasgow Outcome Scale-Extended; $\mathrm{HD}=$ hospital day; $\mathrm{HIC}=$ high-income country; HR = hazard ratio; IQR = interquartile range; KCMC = Kilimanjaro Christian Medical Center; KM = Kaplan-Meier; LMIC = low- and middle-income country; $\mathrm{MOI}=$ mechanism of injury; RTI = road traffic injury; SSA = sub-Saharan Africa; TBI = traumatic brain injury. ACCOMPANYING EDITORIAL DOI: 10.3171/2019.8.FOCUS19682.

SUBMITTED April 24, 2019. ACCEPTED July 31, 2019.

INCLUDE WHEN CITING DOI: 10.3171/2019.7.FOCUS19316. 
and mortality in certain patients with TBI ${ }^{24}$ However, in SSA, a region with an estimated 1 neurosurgeon per 9 million people, hospitals must balance limited surgical capacity with expansive clinical need. ${ }^{11}$ This demands investigation of the surgical impact on TBI outcomes, which can then support triage and surgical intervention decisions. Despite the need, the impact of surgery on TBI outcomes remains unexplored in low-resource settings.

One major cause of in-hospital death for patients with TBI is secondary brain injury resulting from increased intracranial pressure. Neurosurgical decompression, including craniectomies and craniotomies, can relieve elevated intracranial pressure and prevent disability and death in certain TBI patients. TBI morbidity and mortality in SSA remains high due in part to limited neurosurgical resources and a TBI burden that exceeds capacity. In a comparison study, patients with a severe TBI in LMICs had more than twice the odds of death compared to patients in HICs. ${ }^{6}$ TBI surveillance registries from the SSA countries of Tanzania, Malawi, Uganda, and Ethiopia reported mortality rates of $47 \%, 41 \%, 26 \%$, and $21 \%$, respectively, for severe TBI.1,9,22,27 The causes of these mortality rates are multifactorial, including the prehospital and inpatient care settings. Although prehospital quality data in lowresource settings are scarce, the availability of quality inpatient TBI registries from LMICs invites exploration of in-hospital care for patients with TBI in SSA.22

A survival analysis performed using a Cox regression model is a powerful statistical technique to quantify the association between a treatment and outcome for two groups. ${ }^{5}$ For TBI, this technique has potential to measure associations between in-hospital clinical management (e.g., surgical intervention) and outcomes. As of 2010, there were only 12 papers examining TBI management in non-high-income settings, only 1 of which was from a low-income country.,21 Despite a recent increase in published data on TBI management and outcomes in LMICs, $, 10,13,22,27$ there are no previous studies applying survival analyses to inpatient TBI outcomes data.

TBI outcomes data from an area of the world where trauma is an endemic occurrence can help us plan interventions to improve TBI management. This study's objective was to assess the outcomes of patients with TBI at a tertiary referral center in Moshi, Tanzania. Specifically, we wanted to determine if patients with TBI receiving surgery had better outcomes than those not receiving surgery and whether this differs with severity of injury. A greater understanding of the impact of surgery by TBI severity, particularly in a resource-scarce setting, can guide the prioritization of limited resources.

\section{Methods}

\section{Methodological Approach}

In this study we compared the outcomes between patients who received surgical intervention and those who did not by using a survival analysis. A survival analysis is a statistical technique that is well suited for time-to-event data. For calculating the time to event for surgical patients in our study, we could have selected date of admission to outcome or date of surgery to outcome. We tested both options as a sensitivity analysis and found no difference between the two methodological approaches. Because we are interested in the overall health system process and the outcome of patients and not just postsurgical outcomes, we chose date of admission for our time-to-event analysis. One potential confounder with this approach is whether delay to surgery varied by patient Glasgow Coma Scale (GCS) score. We calculated the time to surgery (median, interquartile range [IQR]) for each TBI severity and performed a logistic regression to determine if time to surgery impacted patient outcomes. This variable was not a significant predictor of outcomes, thus supporting our approach.

\section{Study Design}

We conducted a retrospective, cross-sectional observational study. In designing the analysis, we followed the Strengthening the Reporting of Observational Studies in Epidemiology (STROBE) guidelines ${ }^{30}$ and incorporated best-practice recommendations published for time-toevent outcomes and survival analyses..$^{18,23,29}$

\section{Study Setting}

The data for this study were from Kilimanjaro Christian Medical Center (KCMC), a tertiary level hospital in northern Tanzania serving a catchment population of more than 15 million people. KCMC treats approximately 1000 patients with TBI annually. ${ }^{22}$ At the time of data collection, KCMC did not have any physicians who were trained for neurosurgery. General surgery attendings and residents perform all neurosurgical cases. The most common procedures are emergency burr holes or, less frequently, craniectomies/craniotomies for subdural and epidural hematomas. Although burr holes are not the preferred surgical intervention for acute epidural hematomas, this technique may still be used in some lower-resource settings like KCMC. $8,12,14$

In 2013, KCMC established a prospective TBI registry as part of a quality improvement process. ${ }^{22}$ The registry consecutively enrolled all patients presenting to the KCMC Emergency Department for treatment of their acute ( $<24$ hours) TBI. Trained research nurses collected data on the enrolled patients' injury details, acute care, hospitalization care, and condition at discharge. The research team and the study's principal investigator (PI) performed quality analysis of data entering the registry at the following times: 1) at time of data entry and 2) during weekly meetings. ${ }^{22}$

\section{Patient Population}

The study included patients enrolled in the KCMC registry between May 2013 and July 2017. We excluded patients with missing data for the following key variables: admission date, discharge date, received surgery for TBI, and admission GCS score (Fig. 1).

\section{Explanatory Variables}

The analysis included the following variables: age; sex; self-reported consumption of alcohol before the injury; admission GCS score; 25 mechanism of injury (MOI); 


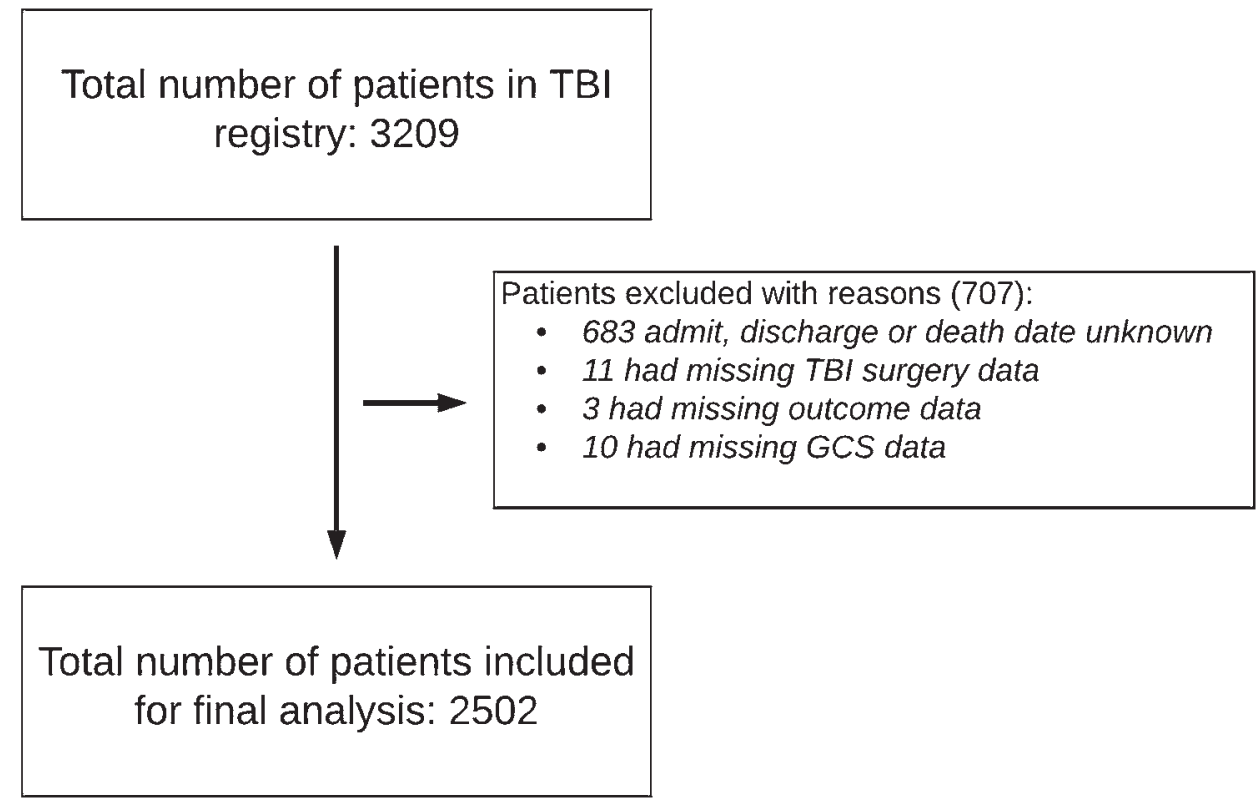

FIG. 1. Diagram illustrating how we arrived at the final study population.

whether the patient had surgery for TBI; and whether the patient was transferred from the surgical ward to the ICU, representing a worsening in their condition or a missed triage from the emergency department. The TBI surgeries variable included mostly emergency burr holes and some craniotomies/craniectomies. The KCMC registry did not include the exact procedure performed.

We stratified patients into 3 groups based on admission GCS score: mild $(14,15)$, moderate $(9-13)$, and severe (3-8) TBI. Because the data included predominantly mild injuries, we used the aforementioned GCS cutoffs to create more balanced subgroups (i.e., mild, moderate, and severe TBIs) for the survival analysis..$^{15}$ We transformed age from a continuous to an ordinal variable with the following categories: $<18,18-29,30-39,40-49$, and $\geq 50$ years. MOIs included road traffic injury (RTI), assault, falls, and other. Examples of other MOIs included accidental trauma to head with object. For patients with mild, moderate, and severe TBI, we also calculated time from admission to surgery. This allowed us to compare delays to surgery by TBI severity and ensure that one group did not have significantly different delays compared to the others. We present these delays to surgery data but did not include them in the analysis, because the primary comparison of this study was outcomes between patients who did not undergo surgery and those who did, not timely versus delayed surgery.

\section{Outcome}

We selected the discharge Glasgow Outcome Scale (GOS) score as the outcome measure for this study (Table 1). The GOS scoring system ranges from 1 to 5, each number representing a different level of recovery as described in Table 1. We dichotomized this variable into good outcome (GOS score of 4-5) and poor outcome (GOS score of 1-3). Approximately halfway through the data collection process, we switched to the Glasgow Outcome Scale-
Extended (GOSE) to improve our descriptions of patients' outcome disabilities. This scale ranges from 1 to 8 . We converted those with a GOSE outcome to a GOS score as shown in Table 1. The registry did not include outcomes after discharge.

\section{Data Analysis}

The data analysis compared the two study groups: patients with TBI receiving (group 1) and not receiving (group 2) surgery. We followed each patient from admission (hospital day [HD] 0) to discharge, inpatient death, or HD 14. We selected HD 14 due to its precedent in the literature to assess acute outcomes for TBI. ${ }^{17,19}$ Additionally, survival plots should continue until $10 \%-20 \%$ of the data remains. ${ }^{18}$ In our study, $13 \%$ of patients remained without an outcome at HD 14. These patients with a poor outcome after HD 14 did not count as an event in the survival analysis (i.e., right censored). We used R Language for Statistical Computing version 3.4.1, including the survminer and tidyverse packages, for data management and statistical analyses. We set significance at a $\mathrm{p}$ value less than 0.05 .

\section{Descriptive Analysis}

We calculated descriptive statistics for demographic and clinical care data, including means, SDs, medians,

TABLE 1. GOS-GOSE conversion

\begin{tabular}{lcc}
\hline \multicolumn{1}{c}{ Outcome } & GOSE Score & GOS Score \\
\hline Death & 1 & 1 \\
\hline Persistent vegetative state & 2 & 2 \\
\hline Severe disability & 3 or 4 & 3 \\
\hline Moderate disability & 5 or 6 & 4 \\
\hline Good recovery & 7 or 8 & 5 \\
\hline
\end{tabular}


and IQR. We used chi-square tests to compare categorical variables.

\section{Kaplan-Meier Survival Plots}

We used the Kaplan-Meier (KM) plots to present the survival curves and test the crude survival between the two study groups. ${ }^{23}$ One KM curve depicted the entire study population and a second set of KM curves depicted patients with mild, moderate, and severe TBI separately. We presented the statistical uncertainty with 95\% CIs (shaded region on plots). Our KM plots travel up with sliding $\mathrm{y}$-axis scales to improve comparison between study groups..$^{18}$ We displayed the number of patients still at risk at a given time below the time axis. The KM plots cannot provide an effect estimate for surgery on outcomes, which led us to build a Cox proportional hazard model (CoxPH).

\section{Cox Proportional Hazard Model}

We built Cox models to examine the association of TBI surgery with outcomes for the overall study population and by TBI severity. The Cox model is a powerful method to analyze survival data, which can produce a hazard ratio (HR; interpreted similarly to an incident rate ratio or relative risk) for poor outcome while controlling for possible confounders. ${ }^{29}$

For appropriate use and inference of a Cox model, the survival curves must be proportional throughout the study period. To assess for proportionality, we 1) checked if the survival curves crossed and 2) used the proportional hazard assumption of a Cox regression test (i.e., CoxPH). If the proportional hazard assumption was violated, we used step functions to add time-interaction terms. ${ }^{26} \mathrm{We}$ used the CoxPH output to determine at which HD to add an interaction term. We produced unadjusted and adjusted effect estimates for surgery.

\section{Institutional Review Board}

We obtained ethical approval from the KCMC Ethics Committee and had an exemption from the Duke University Institutional Review Board because this was a deidentified secondary data analysis of a quality improvement registry.

\section{Results}

The initial cohort comprised all 3209 patients enrolled in the TBI registry. After the exclusion of cases with key data missing, 2502 patients with TBI remained for inclusion in the analysis (Fig. 1).

Of the final 2502 patients, 609 (24\%) received TBI surgery and $1893(76 \%)$ did not receive TBI surgery. There were significantly fewer RTIs and more violent causes of injury in those receiving surgery. Those receiving TBI surgery were also more likely to receive care in the ICU, have a poor outcome, have a moderate or severe TBI, and stay in the hospital longer. There was no significant difference between the two study groups with regard to age, sex, or presence of alcohol (Table 2).

Of the 609 TBI surgeries performed, patients with severe TBI received the highest proportion $(121 / 316,38 \%)$, followed by patients with moderate TBI $(109 / 352,31 \%)$, and those with mild TBI $(379 / 1834,21 \%)$. The median (IQR) for days to surgery was $0.58(0.29,1.35)$. On regression analysis (not shown), time to surgery was not significantly associated with patient outcomes.

Figure 2 is the KM plot for the entire study population. Patients with TBI not receiving surgery (blue line) had more events of poor outcome compared to those receiving surgery. At approximately HD 11 the lines cross, and those receiving surgery had more events of poor outcome.

Figure 3 includes the KM plots for each TBI severity level separately. The slope of the survival curves increases from mild to severe, reflecting an increase in poor outcome rates as severity increases. In each of the 3 plots, the survival curves between patients receiving and not receiving surgery separate initially, converge between HDs 5 and 10, and then separate again. This fluctuation resulted in visible and statistical violations of the proportionality assumption.

The overall study population and populations with severe TBI required time-interaction terms at HDs 3 and 7 to ensure proportionality. Populations with mild and moderate TBI required a time interaction at HD 7 only.

In the unadjusted model (Supplemental Table 1), TBI surgery was associated with a reduced hazard for poor outcome for all study groups before HD 8. This finding was only significant for patients with moderate TBI before HD 8 and patients with severe TBI before HD 4. Surgery was associated with an increased hazard for poor outcome for the overall population with TBI, and for the populations with mild and moderate TBI after HD 7; however, this finding was not significant. In the fully adjusted model (Table 3), surgery was associated with a significantly reduced hazard for poor outcome for all study groups before HD 8. Patients with moderate TBI receiving surgery had the greatest reduction in hazard, an $83 \%$ decrease.

Table 4 provides the Cox model output for the fully adjusted Cox model of the overall study population. Moderate TBI, severe TBI, and transfer from the ward to the ICU significantly increased the hazard for poor outcome. Patients receiving surgical intervention and having an outcome by HD 3 had a $68 \%$ reduction in their HR. Similarly, patients with a surgery and outcome between HDs 4 and 7 had a 54\% reduction in their HR. Time from patient admission to surgery was lowest for those with an outcome between HDs 0 and 3, and highest for those with an outcome between HDs 8 and 14 .

\section{Discussion}

This study is the first analysis of acute TBI outcomes performed using a survival analysis in an LMIC and is one of the largest single-center studies on TBI outcome. Approximately one-quarter of the 2502 patients in our study received surgery for TBI; those who did had a lower hazard for poor outcome than those who did not. To varying degrees, we observed a benefit of surgery for all TBI severities. Surgical intervention was associated with the greatest improvement in outcomes for moderate TBI, followed by mild and severe TBI. The fully adjusted models revealed variables significantly associated with both 
TABLE 2. Descriptive analysis of the variables considered

\begin{tabular}{|c|c|c|c|c|}
\hline \multirow[b]{2}{*}{ Variable } & \multicolumn{3}{|c|}{ Patient Population } & \multirow[b]{2}{*}{$\mathrm{p}$ Value } \\
\hline & All Patients, $N=2502$ & TBI Surgery, $n=609$ & No TBI Surgery, $n=1893$ & \\
\hline Age in yrs & & & & 0.33 \\
\hline$<18$ & $368(15)$ & $98(16)$ & $270(14)$ & \\
\hline $18-29$ & $881(36)$ & $195(32)$ & $686(37)$ & \\
\hline $30-39$ & $554(22)$ & $139(23)$ & $415(22)$ & \\
\hline $40-49$ & $325(13)$ & $87(14)$ & $238(13)$ & \\
\hline$\geq 50$ & $347(14)$ & $87(14)$ & $260(14)$ & \\
\hline Missing & 27 & 3 & 24 & \\
\hline Sex & & & & 0.72 \\
\hline Male & $2084(83)$ & $510(84)$ & $1574(83)$ & \\
\hline Female & $417(17)$ & $98(16)$ & $319(17)$ & \\
\hline Missing & 1 & 1 & 0 & \\
\hline Presence of alcohol & & & & 0.22 \\
\hline Yes & $662(27)$ & $147(24)$ & $515(27)$ & \\
\hline No & $1211(49)$ & $299(49)$ & $912(49)$ & \\
\hline Unknown & $607(24)$ & $160(26)$ & $447(24)$ & \\
\hline Missing & 22 & 3 & 19 & \\
\hline $\mathrm{MOI}$ & & & & $<0.001$ \\
\hline RTI & $1693(68)$ & $339(56)$ & $1354(72)$ & \\
\hline Assault & $362(15)$ & $122(20)$ & $240(13)$ & \\
\hline Fall & $252(10)$ & $72(12)$ & $180(10)$ & \\
\hline Other & $187(8)$ & $71(12)$ & $116(6)$ & \\
\hline Missing & 8 & 5 & 3 & \\
\hline \multicolumn{5}{|l|}{ Surgical ward to ICU } \\
\hline Yes & $357(15)$ & $230(40)$ & $127(7)$ & $<0.001$ \\
\hline No & $2029(85)$ & $338(60)$ & $1691(93)$ & \\
\hline Missing & 116 & 41 & 75 & \\
\hline \multicolumn{5}{|l|}{ Time to surgery } \\
\hline Median days (IQR) & $0.58(0.29-1.35)$ & $0.58(0.29-1.35)^{\star}$ & NA & \\
\hline Outcome & & & & $<0.001$ \\
\hline Poor: GOSE 1-4 or GOS 1-3 & $253(10)$ & $85(14)$ & $168(9)$ & \\
\hline Good: GOSE 5-8 or GOS 4-5 & $2249(90)$ & $524(86)$ & $1725(91)$ & \\
\hline Days to median outcome (IQR) & $3.4(1.8-7.2)$ & $6.2(3.6-11.2)$ & $2.8(1.5-5.7)$ & \\
\hline TBI & & & & $<0.001$ \\
\hline Mild: GCS 14-15 & $1834(73)$ & $379(62)$ & $1455(77)$ & \\
\hline Moderate: GCS 9-13 & $352(14)$ & $109(18)$ & $243(13)$ & \\
\hline Severe: GCS 3-8 & $316(13)$ & $121(20)$ & $195(10)$ & \\
\hline
\end{tabular}

poor and good outcome. This study leveraged advanced statistical methods and a robust data set from an LMIC to investigate the association of surgery with acute TBI outcomes and how that association changes according to TBI severity.

\section{Impact of Surgery}

We found surgery to be associated with a reduced HR for poor outcome for all TBI severity groups. Impressively, given that $\mathrm{KCMC}$ relies on general surgeons and does not have trained neurosurgeons, the positive association of TBI surgery with outcomes is a critical finding for LMICs experiencing shortages of highly skilled professionals. This finding supports the potential of task-sharing neurosurgical emergencies with general surgeons while efforts are ongoing to increase the number of trained neurosurgeons globally. Additionally, patients with moderate TBI who received surgery had the greatest reduction in HR, followed by those with mild and then severe TBI. Our find- 


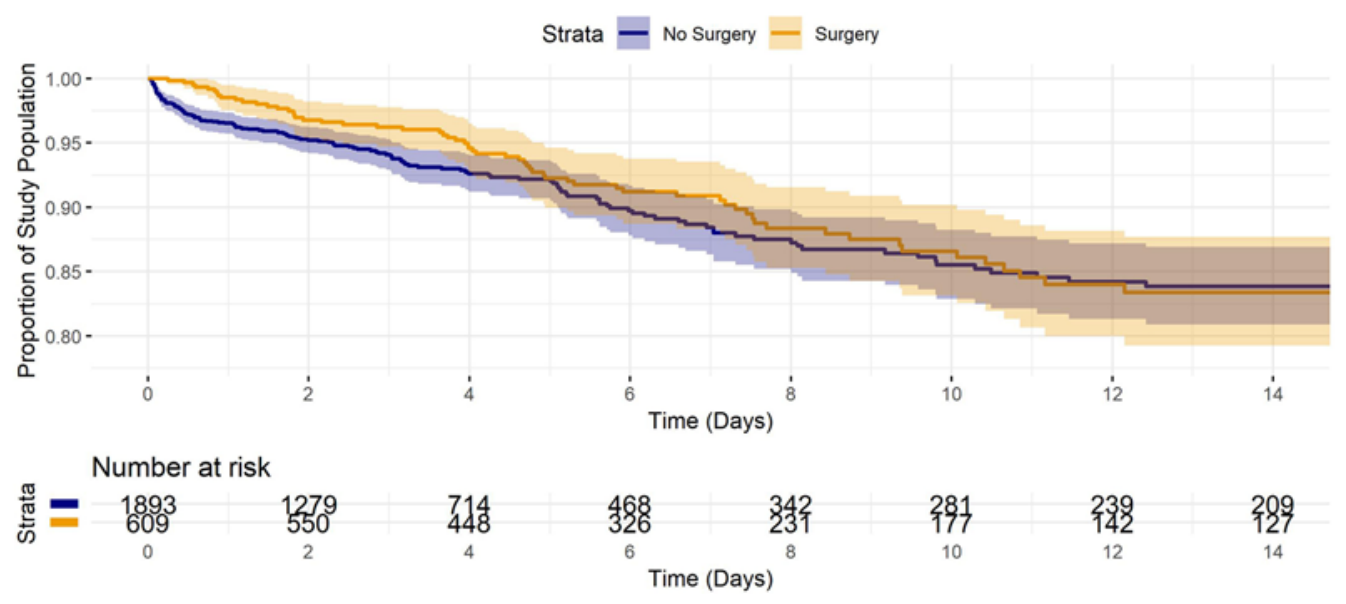

FIG. 2. KM plot comparing outcomes between those receiving and not receiving TBI surgery for the entire study population.

ings, together with previous reports on the overall effects of surgery on patients with severe TBI, should encourage increased attention toward triaging limited resources, especially in regard to focusing resources on those who will receive the greatest impact on their outcome. A retrospective review on postsurgical outcomes of patients with severe TBI found that individuals presenting with a GCS score of 3-5 were nearly 5 times more likely to have a poor outcome at 30 days compared to patients with a GCS score of $6-8 .{ }^{16}$ Cooper et al., in a prospective randomized controlled trial, found that patients with severe TBI receiving decompressive craniectomy compared to the standard level of care had higher odds of unfavorable outcome and similar death rates at 6 months. ${ }^{4}$ These previous findings, along with our data, should encourage research on the reprioritization of care to patients with less severe injuries, a drastic change to the traditional practice in which the most severely injured patients are prioritized for care.

\section{Predictors of Outcomes}

Predictors associated with poor outcome included male sex, patient transfer from surgery ward to ICU, increased age, and moderate and severe TBI. Assault, an intentional cause of TBI, was not significantly associated with poor outcome in our study. However, previous research has found that intentional injuries are a predictor of poor outcome. ${ }^{31}$ The predictor that was associated with improved outcomes was undergoing TBI surgery. This finding was not significant for patients with a discharge past HD 7. The limited number of patients remaining in the study after HD 7 ( $<25 \%$ of the study population) provides a statisti-
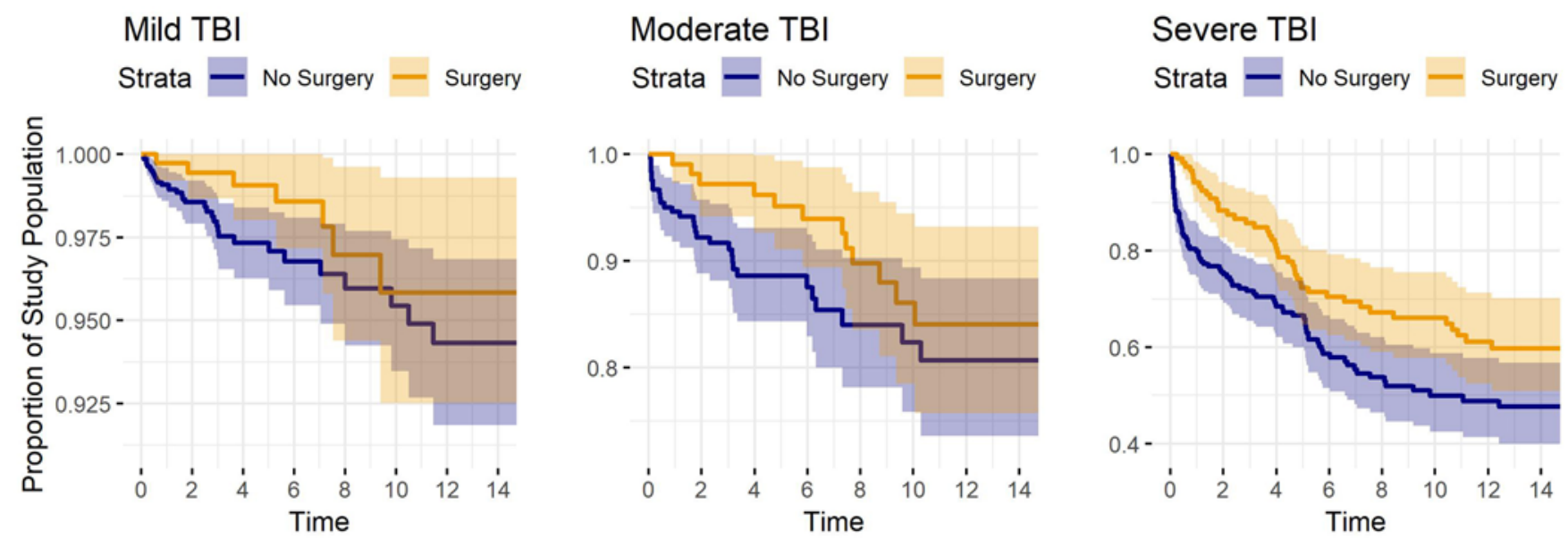

\section{Number at risk}
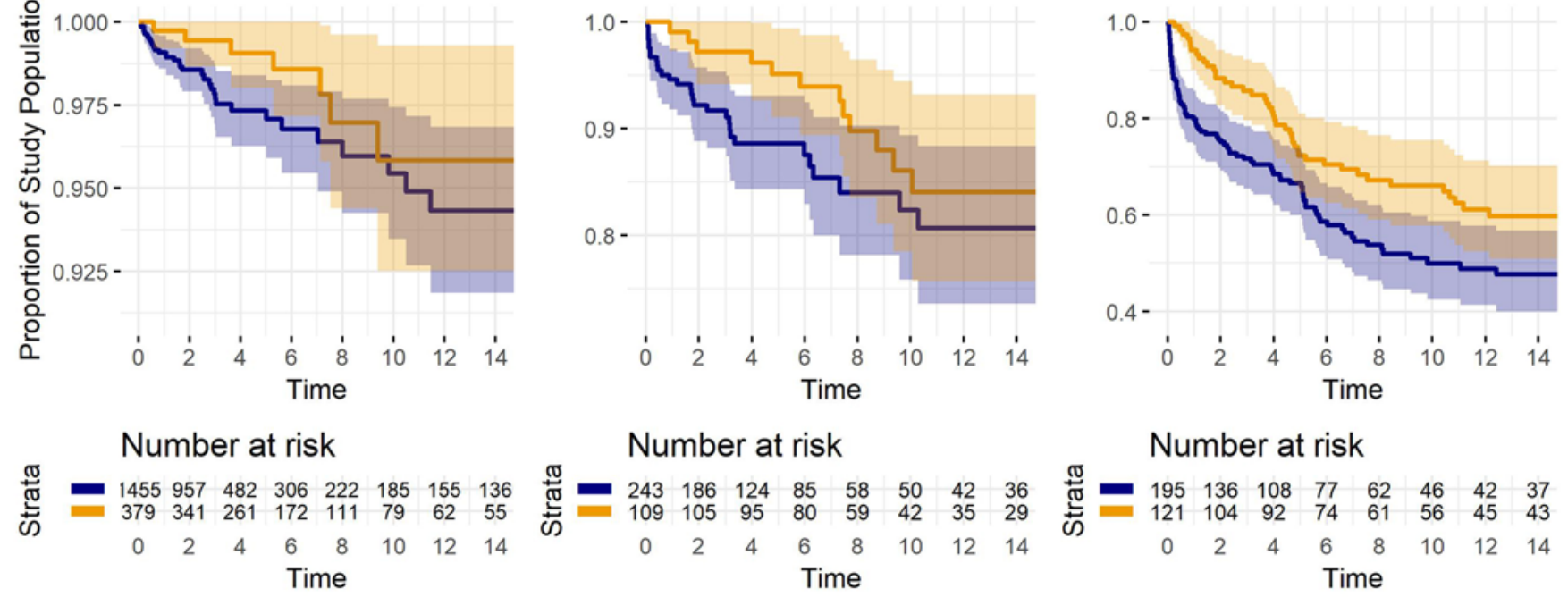

\section{Number at risk}

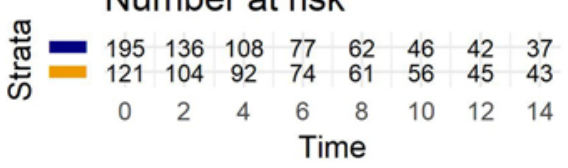

FIG. 3. KM curves comparing outcomes between patients with TBI receiving and not receiving surgery, stratified by GCS severity groups. We used a sliding y-axis scale. 
TABLE 3. Fully adjusted CoxPH for TBI surgery by injury severity with time interactions

\begin{tabular}{|c|c|c|c|c|c|c|c|c|}
\hline \multirow[b]{2}{*}{ HD } & \multicolumn{2}{|l|}{ Overall } & \multicolumn{2}{|l|}{ Mild } & \multicolumn{2}{|c|}{ Moderate } & \multicolumn{2}{|l|}{ Severe } \\
\hline & $\mathrm{HR}(95 \% \mathrm{Cl})$ & $p$ Value & $\mathrm{HR}(95 \% \mathrm{Cl})$ & $p$ Value & $\mathrm{HR}(95 \% \mathrm{Cl})$ & $p$ Value & $\mathrm{HR}(95 \% \mathrm{Cl})$ & $p$ Value \\
\hline $0-3$ & $0.32(0.19-0.54)$ & $<0.001$ & $0.20(0.06-0.64)$ & 0.006 & $0.17(0.06-0.49)$ & $<0.001$ & $0.47(0.24-0.89)$ & 0.02 \\
\hline $4-7$ & $0.46(0.25-0.86)$ & 0.02 & & & & & $0.65(0.30-1.39)$ & 0.26 \\
\hline $8-14$ & $0.77(0.36-1.66)$ & 0.51 & $0.69(0.14-3.31)$ & 0.64 & $1.23(0.23-6.68)$ & 0.81 & $0.70(0.23-2.13)$ & 0.53 \\
\hline
\end{tabular}

Covariates included but not shown are $\mathrm{MOI}$, sex, alcohol, transfer from surgery to ICU, age, and GCS score (for overall only). HDs 0-3 and 4-7 are combined for mild and moderate TBI.

cal explanation. Clinically this finding suggests that those with a later hospital discharge and a longer delay to surgical intervention had worse outcomes. We found those with an outcome before HD 4, a group with the shortest time from admission to surgery, received the greatest benefit from surgery. Although the impact of delays in surgery on outcomes was not the primary objective of this study, this association of delays in care with poor outcomes is well supported by previous research. ${ }^{20,28}$

TABLE 4. Fully adjusted CoxPH results for entire study population

\begin{tabular}{|c|c|c|}
\hline Variable & $\mathrm{HR}(95 \% \mathrm{Cl})$ & p Value \\
\hline \multicolumn{3}{|l|}{ Sex } \\
\hline Male & $1.51(0.98-2.35)$ & 0.06 \\
\hline \multicolumn{3}{|l|}{ Age in yrs } \\
\hline$<18$ & Reference & Reference \\
\hline $18-29$ & $1.91(1.04-3.52)$ & 0.04 \\
\hline $30-39$ & $2.45(1.29-4.64)$ & 0.006 \\
\hline $40-49$ & $2.51(1.28-4.91)$ & 0.007 \\
\hline$\geq 50$ & $1.92(0.99-3.72)$ & 0.05 \\
\hline \multicolumn{3}{|l|}{$\mathrm{MOI}$} \\
\hline RTI & Reference & Reference \\
\hline Assault & $0.73(0.41-1.32)$ & 0.30 \\
\hline Fall & $1.55(0.96-2.51)$ & 0.07 \\
\hline Other & $1.24(0.67-2.28)$ & 0.49 \\
\hline \multicolumn{3}{|l|}{ Alcohol } \\
\hline No & Reference & Reference \\
\hline Yes & $0.90(0.59-1.38)$ & 0.63 \\
\hline Unknown & $1.03(0.70-1.52)$ & 0.88 \\
\hline \multicolumn{3}{|c|}{ TBI surgery (HD of outcome)* } \\
\hline HD 0-3 & $0.32(0.19-0.54)$ & $<0.001$ \\
\hline HD 4-7 & $0.46(0.25-0.86)$ & 0.02 \\
\hline HD 8-14 & $0.77(0.36-1.66)$ & 0.51 \\
\hline \multicolumn{3}{|c|}{ Surgery to ICU transfer } \\
\hline Yes transferred & $3.43(2.32-5.05)$ & $<0.001$ \\
\hline \multicolumn{3}{|l|}{ GCS } \\
\hline Mild & Reference & Reference \\
\hline Moderate & $3.21(1.99-5.17)$ & $<0.001$ \\
\hline Severe & $8.01(5.18-12.4)$ & $<0.001$ \\
\hline
\end{tabular}

* This variable indicates that the patient had surgery and an outcome within the specified time frame. This variable correlates with time to surgery (Supplemental Table 2).

\section{Considerations for Survival Analysis}

The lessons learned by applying survival analysis (including KM plots and a Cox model) to our data have important clinical and statistical considerations. First, our data exhibited significant nonproportionality: the effect of surgery varied depending on the day of hospital discharge and TBI severity. Statistically, this finding required the use of time-interaction terms in the Cox model to avoid erroneous inferences from the data., ${ }^{3,26}$ For example, patients with severe TBI receiving surgery, compared to those with moderate and mild TBI, had the lowest HR when a timeinteraction term was not included. This finding was the opposite of the association seen when we used time-interaction terms. Clinically, the nonproportionality of our data may represent temporal differences in care (i.e., delays in surgery) and/or clinical deterioration for patients with protracted hospital stays. These potential explanations warrant further investigation. For moderate TBI, the positive association of surgery with outcomes decreased at HD 8. This finding was not seen in patients with mild and severe TBI. Patients with a moderate TBI may therefore be more prone to clinical deterioration compared to those with mild and severe TBI, for whom good and poor outcomes, respectively, are more confidently expected. Further research surrounding inpatient care for this specific patient population is necessary to explore this finding. We believe survival analysis techniques, when applied to TBI outcomes, can improve our assessment of inpatient and outpatient clinical care.

\section{Limitations of the Study}

A robust statistical analysis of registry data from a lowresource setting needs careful consideration of limitations. First, the decision to operate or not is influenced by factors that may also contribute to the patient outcome. For example, a patient may not receive surgery due to major comorbidities or other negative prognostic indicators. As a result, a patient suitable for surgery may be better positioned to have a good outcome. To mitigate this limitation, we used the data available to control for predictor of outcome (e.g., GCS score, age). Also, previous research in similar contexts of care has found that limited resources may be focused on more severely injured patients. ${ }^{28}$ The TBI registry used in this study did not include the GCS score at the time of surgery because this information was not always readily available to the research team. The type of surgery was also not documented in the database; however, the general surgery staff endorsed performing mostly burr holes and some hemicraniectomies/craniotomies. The 
registry included fewer patients who suffered severe and moderate TBI than those with mild injuries. The smaller sample size limited our ability to draw inferences from the survival analyses at later HDs. Other than the admission GCS score, we did not have data on the injury itself (e.g., CT results) to understand the severity of the TBI. We also did not have information on other injuries that might have caused poor outcomes. Finally, we did not have patient outcomes data past hospitalization due to the limited postdischarge contact and care in this clinical setting. This limited our analysis to in-hospital outcomes only.

\section{Conclusions}

A detailed understanding of TBI in-hospital outcomes in a low-resource setting is needed to support prudent use of limited, life-saving resources. This paper provides an analysis of surgical management and outcomes data from a tertiary referral center in Moshi, Tanzania. Surgical intervention improved outcomes for all patients with mild, moderate, and severe TBI. The positive impact of surgery to improve outcomes, however, was greatest for patients with moderate and mild TBI. Future research should investigate which patients with TBI are the best candidates for surgery and what factors contribute to clinical deterioration for patients with longer hospital stays, especially in the low-resource setting.

\section{Acknowledgments}

Dr. Staton acknowledges salary support from the Fogarty International Center of the US National Institutes of Health under Award Number K01TW010000 (Principal Investigator, Staton) and the Duke Division of Emergency Medicine.

\section{References}

1. Aenderl I, Gashaw T, Siebeck M, Mutschler W: Head injury-a neglected public health problem: a four-month prospective study at Jimma University Specialized Hospital, Ethiopia. Ethiop J Health Sci 24:27-34, 2014

2. Bagan M: Neurosurgery in Nepal. Surg Neurol 47:512-514, 1997

3. Bellera CA, MacGrogan G, Debled M, de Lara CT, Brouste V, Mathoulin-Pélissier S: Variables with time-varying effects and the Cox model: some statistical concepts illustrated with a prognostic factor study in breast cancer. BMC Med Res Methodol 10:20, 2010

4. Cooper DJ, Rosenfeld JV, Murray L, Arabi YM, Davies AR, D'Urso P, et al: Decompressive craniectomy in diffuse traumatic brain injury. N Engl J Med 364:1493-1502, 2011

5. Cox DR: Regression models and life-tables. J R Stat Soc Series B Stat Methodol 34:187-220, 1972

6. De Silva MJ, Roberts I, Perel P, Edwards P, Kenward MG, Fernandes J, et al: Patient outcome after traumatic brain injury in high-, middle- and low-income countries: analysis of data on 8927 patients in 46 countries. Int J Epidemiol 38:452-458, 2009

7. Dewan MC, Rattani A, Gupta S, Baticulon RE, Hung YC, Punchak M, et al: Estimating the global incidence of traumatic brain injury. J Neurosurg 130:1080-1097, 2019

8. Donovan DJ, Moquin RR, Ecklund JM: Cranial burr holes and emergency craniotomy: review of indications and technique. Mil Med 171:12-19, 2006

9. Eaton J, Hanif AB, Grudziak J, Charles A: Epidemiology, management, and functional outcomes of traumatic brain in- jury in sub-Saharan Africa. World Neurosurg 108:650-655, 2017

10. Eaton J, Hanif AB, Mulima G, Kajombo C, Charles A: Outcomes following exploratory burr holes for traumatic brain injury in a resource poor setting. World Neurosurg 105:257-264, 2017

11. El Khamlichi A: African neurosurgery: current situation, priorities, and needs. Neurosurgery 48:1344-1347, 2001

12. Habibi Z, Meybodi AT, Haji Mirsadeghi SM, Miri SM: Burrhole drainage for the treatment of acute epidural hematoma in coagulopathic patients: a report of eight cases. J Neurotrauma 29:2103-2107, 2012

13. Kuo BJ, Vaca SD, Vissoci JRN, Staton CA, Xu L, Muhumuza $\mathrm{M}$, et al: A prospective neurosurgical registry evaluating the clinical care of traumatic brain injury patients presenting to Mulago National Referral Hospital in Uganda. PLoS One 12:e0182285, 2017

14. Liu JT, Tyan YS, Lee YK, Wang JT: Emergency management of epidural haematoma through burr hole evacuation and drainage. A preliminary report. Acta Neurochir (Wien) 148:313-317, 2006

15. Mena JH, Sanchez AI, Rubiano AM, Peitzman AB, Sperry JL, Gutierrez MI, et al: Effect of the modified Glasgow Coma Scale score criteria for mild traumatic brain injury on mortality prediction: comparing classic and modified Glasgow Coma Scale score model scores of 13. J Trauma 71:11851193, 2011

16. Ogunlade J, Elia C, Duong J, Yanez PJ, Dong F, Wacker MR, et al: Severe traumatic brain injury requiring surgical decompression in the young adult: factors influencing morbidity and mortality-a retrospective analysis. Cureus 10:e3042, 2018

17. Perel P, Arango M, Clayton T, Edwards P, Komolafe E, Poccock S, et al: Predicting outcome after traumatic brain injury: practical prognostic models based on large cohort of international patients. BMJ 336:425-429, 2008

18. Pocock SJ, Clayton TC, Altman DG: Survival plots of timeto-event outcomes in clinical trials: good practice and pitfalls. Lancet 359:1686-1689, 2002

19. Roozenbeek B, Chiu YL, Lingsma HF, Gerber LM, Steyerberg EW, Ghajar J, et al: Predicting 14-day mortality after severe traumatic brain injury: application of the IMPACT models in the brain trauma foundation TBI-trac $\AA$ New York State database. J Neurotrauma 29:1306-1312, 2012

20. Seelig JM, Becker DP, Miller JD, Greenberg RP, Ward JD, Choi SC: Traumatic acute subdural hematoma: major mortality reduction in comatose patients treated within four hours. N Engl J Med 304:1511-1518, 1981

21. Sitsapesan HA, Lawrence TP, Sweasey C, Wester K: Neurotrauma outside the high-income setting: a review of audit and data-collection strategies. World Neurosurg 79:568575,2013

22. Staton CA, Msilanga D, Kiwango G, Vissoci JR, de Andrade L, Lester R, et al: A prospective registry evaluating the epidemiology and clinical care of traumatic brain injury patients presenting to a regional referral hospital in Moshi, Tanzania: challenges and the way forward. Int J Inj Contr Saf Promot 24:69-77, 2017

23. Stel VS, Dekker FW, Tripepi G, Zoccali C, Jager KJ: Survival analysis I: the Kaplan-Meier method. Nephron Clin Pract 119:c83-c88, 2011

24. Stone JL, Lowe RJ, Jonasson O, Baker RJ, Barrett J, Oldershaw JB, et al: Acute subdural hematoma: direct admission to a trauma center yields improved results. J Trauma 26:445450,1986

25. Teasdale G, Jennett B: Assessment of coma and impaired consciousness. A practical scale. Lancet 2:81-84, 1974

26. Therneau T, Crowson C, Atkinson E: Using time dependent covariates and time dependent coefficients in the Cox model. cran.r-project.org (https://cran.r-project.org/web/packages/ 
survival/vignettes/timedep.pdf) [Accessed September 4, 2019]

27. Tran TM, Fuller AT, Kiryabwire J, Mukasa J, Muhumuza M, Ssenyojo H, et al: Distribution and characteristics of severe traumatic brain injury at Mulago National Referral Hospital in Uganda. World Neurosurg 83:269-277, 2015

28. Vaca SD, Kuo BJ, Nickenig Vissoci JR, Staton CA, Xu LW, Muhumuza M, et al: Temporal delays along the neurosurgical care continuum for traumatic brain injury patients at a tertiary care hospital in Kampala, Uganda. Neurosurgery 84:95-103, 2019

29. van Dijk PC, Jager KJ, Zwinderman AH, Zoccali C, Dekker FW: The analysis of survival data in nephrology: basic concepts and methods of Cox regression. Kidney Int 74:705709,2008

30. Vandenbroucke JP, von Elm E, Altman DG, Gøtzsche PC, Mulrow CD, Pocock SJ, et al: Strengthening the Reporting of Observational Studies in Epidemiology (STROBE): explanation and elaboration. Epidemiology 18:805-835, 2007

31. Wagner AK, Sasser HC, Hammond FM, Wiercisiewski D, Alexander J: Intentional traumatic brain injury: epidemiology, risk factors, and associations with injury severity and mortality. J Trauma 49:404-410, 2000

\section{Disclosures}

Dr. Haglund receives support for a non-study-related clinical or research effort that he oversees from NuVasive, UCB Pharma, and Lifenet. He is also a consultant for NuVasive.

\section{Author Contributions}

Conception and design: all authors. Acquisition of data: Sakita, Ndebea, Mmbaga, Staton. Analysis and interpretation of data:

Elahi, Rocha, Nickenig Vissoci. Drafting the article: Elahi, Rocha, da Silva, Haglund. Critically revising the article: all authors.

Reviewed submitted version of manuscript: all authors. Approved the final version of the manuscript on behalf of all authors: Elahi. Statistical analysis: Elahi, Rocha, Nickenig Vissoci. Administrative/technical/material support: Sakita, Ndebea, Fuller, Haglund, Mmbaga, Nickenig Vissoci, Staton. Study supervision: Elahi, Sakita, Ndebea, Fuller, Haglund, Mmbaga, Nickenig Vissoci, Staton.

\section{Supplemental Information}

Online-Only Content

Supplemental material is available online.

Supplemental Tables 1 and 2. https://thejns.org/doi/suppl/10. 3171/2019.7.FOCUS19316.

\section{Correspondence}

Cyrus Elahi: Duke University, Durham, NC. cyrusweal@gmail. com. 